\title{
LA PRAGMÁTICA Y LA ENSEÑANZA DEL ESPAÑOL COMO SEGUNDA LENGUA
}

\author{
Jorge Murillo Medrano \\ Catedrático de la Escuela de Filología, Lingüística y Literatura
}

\begin{abstract}
Recibido 26-IV-2004 • Aceptado 28-IV-2004
\end{abstract}
\begin{abstract}
Resumen: Interesa en este trabajo destacar las interrelaciones entre ciertos conceptos de pragmática lingüistica y el desarrollo de la enseñanza del español como segunda lengua. Se parte de algunas nociones generales de pragmática, como la de actos de habla y la noción de imagen social (face) para dar cuenta de la importancia que reviste integrar estos aspectos en un currículo de español como segunda lengua.

La exposición se divide en tres partes: la primera refiere conceptos generales de pragmalingüística; en la segunda, se reseñan los principales aportes, hasta ahora estudiados, entre esta disciplina y los estudios sobre enseñanza y adquisición de segundas lenguas para, en la tercera, presentar un caso específico sobre el español como L2.
\end{abstract}

Palabras clave: Lingüística Aplicada, Pragmática, Enseñanza de Segundas Lenguas.

\begin{abstract}
The purpose of this study is to point out the interrelationships linking certain concepts of pragmalinguistics and the teaching of Spanish as a second language. Some general notions in pragmatics, such as those concerning speech acts and the face stand out as well as the importance of integrating these aspects within a curriculum of Spanish as a second language.The presentation is divided in three facets: the first one refers to general concepts of pragmalinguistics; in the second one, the most significant contributions so far studied about this discipline and the studies on teaching and the acquisition of second languages are summarized. Lastly, a specific case of L2 Spanish is presented.
\end{abstract}

Key words: Applied Linguistics, Pragmatics, Second Language Teaching.
Conversando un día con una profesora de español como segunda lengua, me comentaba un hecho que le había llamado la atención: un estadounidense, molesto porque no se le había ubicado en un curso que, según él, correspondía con su nivel de conocimiento y manejo de la lengua, había pedido hablar con el coordinador del programa. Al llegar este, lo increpó con las siguientes palabras: Dígame señor, qué pasó aquí, yo quiero saber cómo anda el arroz.

Es evidente que este personaje había aprendido la lengua española en la calle, en la conversación cotidiana, pero ahora que quería ingresar a un instituto de estudio formal no se percataba de que su competencia pragmática solo le daba para las situaciones en las que había aprendido la lengua, pues no sintió la necesidad de adecuar su discurso a los propósitos y participantes de la situación comunicativa del momento.

Es mi interés, en las siguientes páginas, desarrollar un panorama general de la intersección entre la pragmática lingüística y la enseñanza/adquisición del español como segunda lengua, de modo que quede clara la necesidad de incorporar, en nuestras aulas, esta dimensión de la lengua, poco estudiada y valorada al presente. Para ello, dividiré la exposición en tres partes: la primera refiere conceptos generales de pragmalingüística; en la segunda, reseño los principales aportes, hasta ahora 
estudiados, entre esta disciplina y los estudios sobre enseñanza y adquisición de segundas lenguas para, en la tercera, presentar un caso específico sobre el español como L2.

\section{Nociones preliminares de pragmática}

La pragmática, como disciplina dentro de los estudios lingüísticos, no es nueva. Podríamos decir que ha estado presente en las reflexiones sobre el lenguaje desde que estas empezaron a hacerse, aunque su formalización data de las preocupaciones de Charles Morris por incluir al hablante como elemento fundamental de la descripción de los hechos lingüísticos.

No es, tampoco, una corriente que surge, como otras, del seno de la propia lingüística, sino que sus raíces están en campos como la filosofía del lenguaje, con Austin (1962) y su preocupación por la performatividad del lenguaje, y en la sociología (con la etnometodología) y en la antropología misma (con la etnografía). Surge, así, como un campo interdisciplinario y, en la actualidad, se proyecta como una de las perspectivas de estudio más sobresalientes dentro de la lingüística.

Escandell Vidal (1996, 13-14) define la pragmática como "el estudio de los principios que regulan el uso del lenguaje en la comunicación; es decir, las condiciones que determinan tanto el empleo de un enunciado concreto por parte de un hablante concreto en una situación comunicativa concreta, como su interpretación por parte del destinatario". Perfilada de este modo, la pragmática pertenecería a aquellos enfoques lingüísticos de tipo interaccionista (Shriffin, 1994) cuya característica esencial es considerar el lenguaje en su dimensión práctica, de uso, y se opondría a aquellos enfoques estructuralistas en los que se considera a la lengua como una abstracción, un sistema que puede ser solo definido a partir de las relaciones que sostienen unos elementos con otros. Entendida así, la pragmática se ocupa de estudiar el lenguaje en uso, y no cómo se usaría el lenguaje. Toma en cuenta el papel fundamental de los hablantes en la producción lingüística: sus intenciones y las relaciones que establecen por medio de la lengua.

David Crystal (1997) resume muy bien lo anterior al definir a la pragmática como "...the study of language from the point of view of users, especially of the choices they make, the constraints they encounter in using language in social interaction and the effects their use of language has on other participants in the act of communication."1. Con base en lo anterior, entonces, el significado de las palabras o enunciados no estaría dado de antemano, sino que sería el producto de una negociación de los hablantes en el acto comunicativo, negociación en la que entran en juego diversos factores contextuales (espaciotemporales, cognitivos, culturales e históricos) a la vez que cotextuales (lo que se dijo antes y lo que se dirá después). La descripción del hecho lingüístico no se hace, pues, a partir de abstracciones (oraciones gramaticales construidas ad hoc) sino que se recurre al contexto como elemento fundamental para la interpretación del sentido de los enunciados.

Tradicionalmente, los dominios de la pragmática en el estudio del lenguaje han sido los siguientes:

1. Estudio de la deíxis: análisis de todas aquellas formas lingüísticas que solo pueden ser explicadas a partir del contexto o, en otras palabras, las formas que codifican factores contextuales dentro de la lengua: pronombres personales, por ejemplo, formas de tratamiento, adverbios temporales y locativos y la deixis discursiva.

2. Estudio de los actos de habla: se planteó ya cómo Austin y su teoría de los actos de habla constituye un pilar fundamental para los estudios pragmáticos. Para este estudioso, el 
lenguaje es acción y, por lo tanto, los enunciados son actos de habla. A estos enunciados no se les puede someter a la prueba de verdad (es decir, si son verdaderos o falsos) sino que deben ser evaluados en tanto hacen: la emisión de todo enunciado, de acuerdo con este filósofo, tiene siempre tres facetas: lo locutivo (la emisión misma), lo ilocutivo (la fuerza o intención que tiene el hablante al emitirlo) y lo perlocutivo (los efectos que el enunciado produzca en el oyente). Tratando de encontrar una correspondencia entre fuerza ilocutiva y forma lingüística, Searle (1975) se da a la tarea de plantear una clasificación de los distintos tipos de actos de habla según la intención del hablante. Pero tropieza con el hecho de que, en muchas ocasiones, esta forma lingüística no necesariamente se correlaciona con la fuerza ilocutiva: es el caso clásico de la pregunta ¿Podés pasarme la sal?, dicho mientras comemos, a otro individuo presente a la mesa, en que la fuerza ilocutiva (pregunta) esconde otra, más importante, la imperativa. De esta manera, concluye Searle que, en estos casos, estamos frente a actos de habla indirectos; es decir, enunciados cuya forma lingüística no se corresponde necesariamente con la fuerza ilocutiva esperada. Los aportes de Searle son indiscutibles para el estudio del lenguaje desde una dimensión pragmática, como se verá más adelante.

3. Grice, el principio de cooperación y las máximas conversacionales: Grice (1975) es el primero que se sitúa propiamente dentro de la pragmática para hacer sus observaciones. Para él, existe un conjunto de principios que rigen los intercambios conversacionales, los cuales subsume en el llamado principio de cooperación: “Podríamos, entonces, formular un principio general, que es el que se supone que observan los participantes: haga que su contribución a la conversación sea, en cada momento, la requerida por el propósito o la dirección del intercambio comunicativo en el que usted está involucrado." Este principio (que no es normativo) se subdivide en otras normas menores a las que Grice denomina máximas de cantidad ( $\mathrm{Su}$ contribución debe ser todo lo informativa que requiera el propósito del intercambio comunicativo, pero no más de lo requerido), de cualidad (No diga algo que crea falso o de lo cual no tenga pruebas), de relación (Diga cosas relevantes) y de modalidad o modo (Evite la oscuridad y la ambigüedad, sea breve y ordenado). La violación de las máximas supone, para Grice, que el hablante quiere comunicar algo más de lo que dice: surge, así, la noción de implicatura como el excedente de significado que cualquier enunciado puede tener en la conversación: lo que se dice corresponde al contenido proposicional del enunciado mientras que lo que se comunica es lo implicado, la significación adicional. Por ejemplo, en el enunciado $A l$ fin se graduó, además del contenido proposicional básico (terminó finalmente su carrera) el hablante comunica una opinión suya respecto de ese contenido (se le hizo difícil graduarse, tomó más tiempo del necesario, etc...).

4. El estudio de la cortesía: El porqué se dan en la conversación actos de habla indirectos e implicaturas puede encontrar su justificación en los estudios de lo que constituye la cortesía verbal, dominio central en los trabajos de pragmática. La noción de cortesía aparece ya en las preocupaciones de R. Lakoff (1973), cuyo propósito es establecer ciertas reglas pragmáticas que deben regir a los hablantes en la 
conversación: sea claro y sea cortés. La segunda, tiene tres facetas: No se imponga, ofrezca opciones y refuerce los lazos de camaradería. Según Escandell Vidal (1996, 142-143) cada una de estas reglas presupone una situación comunicativa diferente: en la primera (No se imponga) el escenario imaginado sería el de hablantes que están en una relación asimétrica y con una distancia social considerable. La segunda (ofrezca opciones) "se aplica sobre todo cuando hay equilibrio social entre los interlocutores, pero falta familiaridad y confianza" y la tercera (refuerce los lazos de camaradería) parte del supuesto de que existe, entre los interlocutores, una relación cercana de familiaridad 0 amistad. Sin embargo, es el modelo de Brown y Levinson (1987) el que ha logrado una mayor aceptación y aplicabilidad en el estudio de la cortesía lingüística. Ellos parten de que existe, para todos los hablantes, una imagen social ("face") la cual negocian y protegen en el intercambio comunicativo. Según este modelo, cada hablante posee una imagen social con dos componentes básicos: la imagen social positiva y la imagen social negativa, las cuales definen como sigue: "Central to our model is a highly abstract notion of "face" which consists of two specific kinds of desire ("face wants") attributed by interactants to one another: the desire to be unimpeded in one's actions (negative face), and the desire (in some respects) to be approved of (positive face). ${ }^{2}$ " Postulan este concepto como universal y a los comportamientos de los individuos, tendientes a satisfacer esta imagen, los denominan "face work" (actividades de imagen). Sobre esta base, describen una serie de estrategias que los hablantes ponemos en práctica para atenuar la amenaza implícita a la imagen negativa que suponen ciertos actos verbales, especialmente las órdenes y las aserciones. Por otra parte, también describen cuáles son las estrategias típicas de refuerzo a la imagen positiva, las cuales se concretan, generalmente, en actos de habla como el saludo, el cumplido y el agradecimiento. Habría, además, formas de evitar actos típicamente amenazadores de la imagen negativa del otro, los cuales son necesarios en las interacciones verbales de los hablantes: los llamados actos indirectos de habla, en los cuales se comunica por medio de implicaturas. Este tipo de actos revisten suma importancia pues reflejan la concepción que una comunidad lingüística tiene de lo que es ser cortés verbalmente ya que, por medio de ellos, se evidencian estrategias que los hablantes emplean para no dañar la imagen del otro. En última instancia, constituyen un elemento fundamental para deslindar lo que una comunidad de hablantes considera como su identidad en términos del uso del lenguaje ${ }^{3}$.

Haverkate (1994) hace un recuento detallado de todas las estrategias que utilizamos los hablantes para atenuar actos de habla típicamente amenazadores (como las órdenes y las aserciones) y describe, además, los principios de cortesía positiva que rigen los actos de habla no amenazadores de la imagen del otro (como los cumplidos, los agradecimientos o las felicitaciones).

\section{La pragmática y la investigación sobre enseñanza/adquisición de segundas lenguas}

Baste revisar algunos manuales de adquisición/enseñanza de segundas lenguas $^{4}$, para comprobar que el enfoque pragmático ha sido, sino olvidado en su totalidad, 
por lo menos relegado a un segundo plano. Componentes de la lengua como el fonético, el morfosintáctico o el léxico han recibido la mayor parte de la atención, dejando poco espacio para las reflexiones de corte pragmático. Y esto no es de extrañar, en tanto las propuestas metodológicas para enseñar segundas lenguas se han centrado, sobre todo, en los componentes gramaticales (el llamado método tradicional, cuyo objetivo consiste en enseñar la lengua a partir de las estructuras gramaticales, método por lo demás muy común hasta nuestros días), en la pronunciación (el método audiolingüístico) o en la creencia de que, si se adquiere el vocabulario, se domina una segunda lengua. Es decir, manejar una segunda lengua (y por ende enseñarla) supone conocer los componentes gramaticales, fonéticos y léxicos.

Sin embargo, encontramos ya en Sandra Savignon (1983), la idea de que, además de la competencia gramatical, los aprendices de segundas lenguas deben aprender la competencia comunicativa. $\mathrm{Pa}$ ra ella, la competencia comunicativa es un concepto dinámico ( más que estático) y depende de la negociación del significado que se lleve a cabo entre los hablantes, además de que está supeditada al contexto específico de comunicación. También señala que esta competencia comunicativa es relativa, en tanto depende de la cooperación de los participantes en el acto comunicativo.

A partir de las ideas de Savignon, se ha venido insistiendo en la necesidad del estudio y enseñanza del componente pragmático en las clases de segundas lenguas. Kasper y Rose (2002) resumen muy bien todo lo que hasta la fecha se ha llevado a cabo en torno al tema. Parten de una aclaración fundamental en cuanto a que, cuando se habla de estudios de adquisición de pragmática en la interlengua, se ha hecho referencia a dos aspectos diferentes: el primero apunta al estudio del uso de una segunda lengua; es decir, a cómo un hablante no nativo del español, por ejemplo, comprende y produce esa lengua. El segundo se refiere al estudio del aprendizaje de una segunda lengua: en este caso importa cómo los aprendices de una L2 desarrollan la habilidad para entender esa segunda lengua y desenvolverse en ella. Según estos autores, el primer enfoque ha recibido mucha más atención que el segundo, el cual, desde su punto de vista, es el que debe ser investigado con mayor profundidad: solo conociendo cómo los hablantes de una L2 desarrollan la habilidad pragmática, se podrá llegar a comprender mejor el uso que hacen los aprendices de las diferentes estrategias comunicativas.

Es evidente que los aprendices de español como segunda lengua (y aquí vuelvo a la distinción tradicional entre español como L2 y español como lengua extranjera) tienen una doble vía para acceder (adquirir/aprender) los conceptos pragmáticos: por un lado podrían hacerlo en el contacto diario con hablantes nativos, lo cual se ha denominado como programa no intervencionista $y$, por el otro, existe la posibilidad de que le sean enseñados estos conceptos en el aula (programa intervencionista). Esto nos lleva a la pregunta de si sería suficiente la exposición natural a una segunda lengua para llegar a tener una competencia comunicativa total o si debe intervenir el profesor de L2 con un programa específico para este fin. Schmidt (1993) aboga por la segunda alternativa pues considera que las funciones pragmáticas y los factores contextuales a menudo no son tan evidentes para los aprendices en un medio natural, aun cuando hayan pasado largo tiempo viviendo en la cultura meta (Recuérdese el ejemplo de la introducción). Bardovi-Harlig (2001), por su parte, luego de un estudio, concluye que es necesario enseñar la pragmática en el aula, pues los aprendices que no pasan por este proceso difieren significativamente de los hablantes nativos en su capacidad y desarrollo pragmáticos.

¿Qué significaría diseñar un programa en el que se incluya el componente 
pragmático de la lengua como uno de los que se debe enseñar explícitamente?

Como condición sine qua non para la elaboración de un programa de esta índole, debe pensarse en una teoría sobre la adquisición del lenguaje que dé cuenta de cómo se adquiere (si es que se adquiere) la dimensión pragmática de la lengua, especialmente del español. Hasta ahora, sin embargo, hay, como lo señalan Kasper y Rose (2002) pocos estudios en esta dirección. Ellos se dan a la tarea de inventariar lo hecho hasta el 2002 y concluyen que los estudios son escasos y aislados y, para nuestros propósitos, no encuentran ninguno cuya lengua meta sea el español.

El segundo aspecto que debe considerarse es si, en efecto, es enseñable la pragmática y, si lo es, cuáles son los enfoques metodológicos que más propician el aprendizaje de esta dimensión de la lengua. De nuevo, Kasper y Rose (2002, 269) evalúan varios estudios hechos al respecto, y llegan a la conclusión de que “...there is considerable evidence indicating that a range of features of second language pragmatics are teachable. These include a variety of discoursal, pragmatic, and sociolinguistic target of instruction, such a discourse markers and strategies, pragmatic routines, speech acts, overall discourse characteristics, and pragmatic comprehension. Second, it appears that learners who receive instruction fare better than those who do not." "

Lo anterior nos llevaría a concluir que, aunque existen algunos aspectos pragmáticos de la lengua que son enseñables y que ciertos enfoques de enseñanza son más aptos para esta tarea (especialmente aquellos que se centran en el significado, como los enfoques comunicativos, el programa nocional-funcional y la sugestopedia, por ejemplo), existe la necesidad de llevar a cabo una investigación rigurosa que permita determinar, para el español, cuáles son los aspectos pragmáticos que son más susceptibles de ser enseñados, en qué nivel del aprendizaje y cómo se puede hacer (es decir, cuáles son los enfoques metodológicos más apropiados para tal cometido). Debe tomarse en cuenta, además, que los que enseñamos español como L2 en Costa Rica, tendríamos que considerar factores externos al aula como posibles influencias en la adquisición de la competencia pragmática $y$, por lo tanto, las investigaciones al respecto deben extremar las medidas para que los resultados no se vean sesgados por variables extra clase.

Debe dejarse en claro, por otra parte, que cuando se propone un programa de enseñanza a partir del enfoque pragmático no se está negando la necesidad de hacerlo tomando en cuenta los otros componentes de la lengua: el fonético-fonológico, el morfosintáctico y léxico-semántico, pues lo pragmático no siempre puede desligarse de ellos y, más bien, los permea en su totalidad. Muchos de los recursos pragmáticos de los que echamos mano los hablantes de la lengua están ya codificados en la gramática y, cuando no lo están, nos valemos de diferentes recursos lingüísticos para lograr nuestros propósitos comunicativos.

\section{Hacia una enseñanza del español como L2 que incluya lo pragmático}

Si bien es cierto es innegable que el convivir con hablantes nativos en la cultura meta facilita el que un aprendiz de español como L2 adquiera nociones pragmáticas (pues evidentemente tendrá mucho input disponible), es mi opinión que también el profesor debe procurar, en la clase, integrar los aspectos pragmáticos en su currículo. Para hacerlo, podría tomar dos caminos: o lo integra de manera implícita, es decir, a lo largo de los ejercicios o tareas programadas, o bien lo hace de forma explícita (como reflexión metapragmática). $\mathrm{O}$ una tercera posibilidad es tratar de hacerlo de ambas maneras, tomando en cuenta el nivel de los 
estudiantes y, especialmente, la dificultad del concepto o aspecto por estudiar. Por ejemplo, sería casi imposible hacer reflexión metapragmática, en el aula, con estudiantes de nivel principiante y, en ese caso, lo mejor sería hacerlo de manera implícita. Por el contrario, con los avanzados se posibilita la explicación consciente del fenómeno y además es conveniente hacerlo.

Otro aspecto que debe considerarse es qué elementos de la pragmática de la lengua pueden ser enseñados en los distintos niveles, tal y como lo planteamos arriba. La respuesta no es definitiva, pero sí, por ejemplo, es definitivo que ciertos actos de habla (como el saludo, la despedida, las peticiones corteses y las disculpas), deberían programarse ya para un nivel inicial no importa si las estructuras gramaticales necesarias para emitir estos actos de habla se estudian o no en ese nivel. Aunque ciertos métodos como el tradicional de gramática-traducción no permitirían esto, otros como los asociados al enfoque comunicativo o al programa nocional-funcional serían más propensos a ser usados con este propósito (se enfocan en la negociación de significados).

El problema principal radica en que, para lograr un programa como el anterior, es necesario investigar cómo funcionan los aspectos pragmáticos del español en general (para lo cual hay algunos estudios, no muchos) y del español hablado en Costa Rica en particular (y a este respecto los trabajos hechos realmente son muy escasos). Debido a mi interés particular en este tema, he venido ejecutando, desde hace tres años, un proyecto de investigación sobre aspectos pragmáticos del español de Costa Rica y, aunque me he centrado en aspectos relacionados con la cortesía ver$\mathrm{bal}^{6} \mathrm{y}$ formas de atenuación en la conversación coloquial, creo que esto puede arrojar luces sobre cómo funcionan estas categorías en nuestro medio, y los resultados de la investigación servirían también de base para propuestas curriculares no solo en la enseñanza del español como lengua materna sino también, y muy especialmente, del español como segunda lengua.

La primera etapa del proyecto ha consistido en la indagación metapragmática (por medio de cuestionarios ${ }^{7}$ ) en los que se plantean ciertas situaciones hipotéticas y se les pide a los informantes que respondan, de manera consciente, qué dirían en esos contextos particulares. Claro está que estos datos solo pueden tomarse como indicadores imaginarios de situaciones reales, pero este tipo de encuestas (llamadas de hábitos sociales ${ }^{8}$ ), proporcionan información valiosa sobre el comportamiento verbal de una comunidad de habla.

G. Piatti $(2003,204)$ ha utilizado estos tests para la investigación de enseñanza de español como segunda lengua en vista de que “...cuando estas pruebas se hacen a los extranjeros, en la investigación del aspecto pragmático de la interlengua, podemos extraer información sobre el conocimiento de estos hablantes y su actuación bajo condiciones más o menos exigentes de encuentros conversacionales pautados y simulados." Con base en la experiencia de esta investigadora, decidí aplicar el cuestionario a un grupo de estudiantes avanzados, como un primer acercamiento, para valorar cuáles serían las estrategias de cortesía usadas por estos hablantes en ciertas situaciones de comunicación que supondrían una amenaza a la imagen del otro (peticiones o consejos) y en las cuales esperaríamos que los hablantes desplegaran un cúmulo de estrategias atenuadoras.

Los estudiantes eran todos del nivel avanzado (de acuerdo con los criterios internos del Instituto en el que estudiaban, los cuales se corresponden con los de ACTFL) y algunos habían vivido en países de habla hispana (semanas, meses y hasta años) mientras que, para otros, esta era su primera experiencia en un país donde se hablara la lengua meta. Todos los estudiantes eran de origen estadounidense y mayores de edad y aprendían la lengua con un propósito específico: servir como misioneros 
de una orden religiosa en América Latina. Se les pidió que respondieran lo más espontáneamente posible y todo el cuestionario estaba en español y debía, por supuesto, ser contestado en esa misma lengua. De los resultados, solo tomo dos situaciones de la segunda parte, pues el tiempo me impediría comentar todas las respuestas; las seleccionadas son las siguientes:

1. Usted necesita que su profesor le dé más tiempo para la realización de un trabajo. Escriba lo que le diría exactamente.

2. Su amiga Cintia ha engordado mucho y usted quiere advertirla. Escriba lo que le diría exactamente.

En la primera situación, se da una relación asimétrica y con bastante distancia social y el acto de habla es un pedido, mientras que en la segunda la relación es de solidaridad, con menos distancia social y el acto de habla es un consejo. A pesar de las diferencias en cuanto al grado de imposición y la distancia social, ambos actos de habla resultan amenazadores de la imagen del otro, especialmente el segundo pues invade la esfera íntima de Cintia, además de que opinar sobre la apariencia física de los otros es un asunto muy delicado, aun en ámbitos familiares. Se esperaría, en consecuencia, que los hablantes usaran una serie de atenuaciones para mitigar el efecto amenazador de estos actos de habla. Transcribo a continuación algunas de las respuestas dadas por los estudiantes. Para la primera situación tenemos:

(1) Perdón, pero no he terminado con mi trabajo. ¿Es posible que tenga más tiempo para completarlo?

(2) Profesor, ¿me daría más tiempo para la realización de mi trabajo?

(3) Profesor, por favor, ¿me daría más tiempo para realizar este trabajo? Lo siento que soy muy atrasado.
(4) Había problemas con este trabajo, ¿podría darnos más tiempo para realizar?

(5) Todavía no he podido hacer el trabajo. ¿Podría darme más tiempo, por favor?

(6) Por favor, ¿podría tener más tiempo para esta tarea?

(7) Lo siento que no he tenido tiempo para terminar el trabajo. Le ruego, profe, que me regale un poquito más de tiempo para hacerlo bien.

(8) Profesor, disculpe. Tengo una situación difícil. Sé que es muy importante entregar los trabajos a tiempo y me da mucha pena pedirle esto, pero tengo una situación muy especial en la casa y quería saber si usted me podría autorizar un tiempito más para terminar el trabajo que nos asignó.

Aparte de algunos problemas relacionados con la gramática, las diferencias en la competencia pragmática de estos aprendices varía sustancialmente de unos a otros. En todas ellas, sin embargo, hay uso de la pregunta como mecanismo para mitigar los efectos de la petición (que supone amenaza a la imagen del profesor). En relación con actos de habla preparatorios para la petición o ruego, solamente cinco de ellos [(1), (4),(5),(7) y (8)] los utilizan, en unos casos más elaborados que en otros. En (2), solo se usa la forma de tratamiento, mientras que en (3) se emplea la fórmula cortés por favor, al igual que en (6), además de una justificación pos-petición.

El uso del condicional de cortesía es otra de las estrategias utilizadas por los aprendices, excepto en (1) en cuyo lugar el hablante prefiere la estrategia de la desfocalización por medio de la estructura impersonal, lo cual hace probablemente que evaluemos, como hablantes nativos, esa petición como la menos efectiva pragmáticamente.

Deliberamente he dejado al final los ejemplos que considero como más cercanos a lo que diría un hablante nativo en esa 
situación. No obstante, en (7), hay algo que no calza: es un problema de transferencia pragmática al usar la frase "I am sorry" como equivalente a "Disculpe". El resto de la petición de (7) despliega tres estrategias atenuadoras más, típicas del habla costarricense, que dan a la petición un carácter más natural: la forma abreviada (profe), el verbo regalar y el diminutivo. En (8), prácticamente se reúnen todas las posibilidades de atenuación que uno, como hablante nativo, podría esperar y que se dan, solo fragmentariamente en los otros casos.

Como última observación pertinente al respecto de estos ejemplos, solo resta indicar que (8) es una respuesta dada por una mujer que ha estudiado español por aproximadamente siete años y que solamente ha vivido en países de habla hispana por siete meses (a diferencia por ejemplo de quien proporciona la respuesta (1), que había estudiado español por 4 años y había vivido por igual número de años en México ${ }^{9}$.

En el caso de la segunda situación planteada, algunas de las respuestas son las siguientes:

(1) Cintia, tengo miedo de que usted vaya a tener daño a su salud. Yo también quisiera bajar el peso. Quizá podamos reunir cada semana para compartir recetas de baja calorías, etc..

(2) Cintia, estás muy cariñosa a mí, por eso tengo que decirte que estoy muy preocupada por tu salud...

(3) Cintia, no debería comer tan mucho.

(4) Cintia, estoy tratando de perder el peso. ¿Quisiera ir conmigo para correr o hacer ejercicio?

(5) ¿Podríamos ir al parque para divertirnos?

(6) "Yo no voy a decir nada en realidad, siempre y cuando ella no me pregunte".
(7) Cintia, he querido por mucho tiempo empezar a caminar un ratito por las mañanas - es muy bueno para la salud. ¿Quieres acompañarme?

(8) Hola, Cintia, ¿cómo estás?... Yo, también, gracias, y en la casa también. Vieras que mi compañera está haciendo dieta y le está funcionando super bien. Yo te la puedo conseguir, por si te interesa. Hay dietas y dietas, ¿no? Pero parece que esta sí funciona.

Debido al alto grado de amenaza que supone un consejo de este tipo, era de esperarse, por un lado, que los estudiantes trataran de desplegar un mayor número de estrategias atenuadoras o que, en su defecto, como lo hace (6) exprese que no diría nada. Otra variable que podría incidir aquí es el sexo: las respuestas (3), (4) y (5) fueron dadas por hombres; el resto por mujeres

Según Haverkate (1994), los consejos se ubican dentro del ámbito general de las exhortaciones no impositivas: "El hablante no impositivo, en cambio, procura conseguir que el oyente realice el acto exhortado primariamente en beneficio de sí mismo. Los principales componentes de esta clase son el consejo, la recomendación y la instrucción" (p. 148). Son intrusiones en el dominio personal de los otros y, por ende, constituyen actos de habla que amenazan la imagen negativa del interlocutor.

El mecanismo general de atenuación de los ejemplos anteriores es la inclusión del "yo" como supuesto beneficiario también del consejo dado a Cintia: se establece así un lazo de solidaridad con el otro que mitiga, en parte, la amenaza del acto de habla. La estrategia es presentar el acto como una amenaza, entonces, no solo para Cintia, sino para quien habla también, de modo que se evidencie un interés común por el problema de la gordura. Aparecen, por ejemplo, en (1), (4) y (7) enunciados en primera persona como actos de habla preparatorios y en (5) se emplea la primera persona 
plural. El hablante de (8) prefirió recurrir a la desfocalización total (se refiere a un tercero) y echa mano del ejemplo como mecanismo para dar el consejo o, e como en (7), en que se presenta una aseveración general para argumentar a favor del consejo (es bueno para la salud).

Otros mecanismos atenuadores empleados son el uso del condicional y del imperfecto de subjuntivo como formas de proyectar el eje deíctico modal temporal hacia otro momento diferente del presente de la enunciación. El uso de estructuras condicionales, como en (8), o del adverbio de duda como en (1) enmascaran enunciados deónticos como si fueran epistémicos.

En cuanto a la efectividad pragmática del consejo, notamos que (3), por ejemplo, es demasiado directo (solo usa un atenuador, el condicional) y, al igual que (4) y (5), las respuestas de los otros hombres, es lacónico. Desde mi punto de vista, aunque (1) utilice ciertas estrategias eficaces, el acto preparatorio (tengo miedo de que usted vaya a tener daño a su salud) es totalmente "infeliz", en términos de Austin.

De nuevo (7) y (8) son las respuestas que, a mi parecer, se acercarían más a lo que un hablante nativo diría ${ }^{10}$. Utilizan mecanismos de desfocalización deíctica personal, además de otros recursos pragmagramáticos que la lengua ofrece.

De este modo, puede verse que la competencia pragmática de estos hablantes varía aunque, en principio, todos deberían evidenciar un nivel parecido a este respecto pues están en un nivel avanzado. Pero, precisamente, ese es el problema: estar en un nivel avanzado de estudio del español (según criterios de ubicación generales y los mismos de ACTFL) supone manejar ciertas estructuras gramaticales, fonéticas y léxicas en las cuatro habilidades (leer, escuchar, hablar y escribir), pero no necesariamente en el nivel pragmático, pues estas normalmente no son evaluadas para ubicar a los estudiantes.

\section{A manera de conclusión}

Partiendo de nociones generales de pragmática (como actos de habla e imagen social), se ha evaluado la pertinencia de incluir, en un currículo de español como L2, aspectos relacionados con la pragmática de la lengua. Como evidencia para lo anterior, se comentaron estudios que se han hecho sobre este tema en un nivel más general. Se analizaron, por otra parte, datos obtenidos de estudiantes avanzados para demostrar que carecen de entrenamiento en el uso de estrategias pragmática en la conversación.

El propósito fundamental de estas reflexiones no era plantear un modelo metodológico para la enseñanza de la pragmática en las clases de español como L2 sino, más bien, despertar el interés y motivar a aquellos que trabajan en este campo a realizar investigaciones que puedan aclarar y guiar, con mayor certeza, cómo incluir todos estos aspectos pragmáticos en la enseñanza del español como segunda lengua.

\section{Notas}

1. "El estudio del lenguaje desde el punto de vista de los usuarios, especialmente de las selecciones que hacen, de las restricciones que encuentran al utilizar el lenguaje en la interacción social y de los efectos que el uso del lenguaje tiene en los otros participantes del acto comunicativo." (Traducción del autor).

2. "Medular en nuestro modelo es una noción muy abstracta de "imagen social" la cual está constituida por dos tipos específicos de deseos (deseos de imagen social) que se atribuyen los interactuantes: el deseo de no ver amenazadas las acciones propias (imagen social negativa), y el deseo (en cierto sentido) de ser aprobado (imagen social positiva)." (Traducción del autor).

3. Existe una serie de estudios que ha puesto en evidencia el etnocentrismo y la falta de supuesta universalidad del modelo de Brown y Levinson. Al respecto, pueden consultarse 
Matsumoto (1988), Mao (1994), Frazer (1990) y Bravo (1999), entre los varios autores que hacen críticas al modelo B/L.

4. He revisado algunos manuales tanto de adquisición (Harper, Jeremy (1983); Klein, Wolfgang (1986); Rivers, Acevedo y Heflin (1994); Omaggio, A. (1993); Larsen Freeman, D. y Long, M. (1994); Cook, V. (2001), Sánchez, A. (1997) y Srum, J. y Glisan, E. (1994).

5. "Existe una considerable evidencia que indica que ciertas características pragmáticas de las segundas lenguas se pueden enseñar. Entre estos hay una variedad de aspectos discursivos, pragmáticos y sociolingüísticos de la instrucción, tales como marcadores y estrategias discursivas, rutinas pragmáticas, actos de habla, características discursivas generales y comprensión gramática. Además, parece que aquellos aprendices que reciben instrucción tienen mejores resultados que aquéllos que no." (Traducción del autor).

6. Veáse el artículo "La cortesía verbal en el español de Costa Rica”. (2002).

7. Se sigue el modelo propuesto por Kasper, (2000, 326), (production questionnaires), descritos como una de las formas de recopilación de datos pragmáticos.

8. El modelo, planteado originalmente por M. Sifianou (2001), ha sido aplicado por Nieves Hernández (2003) en España y por Murillo (2003), en Costa Rica.

9. Esto podría indicar que factores como la instrucción y el tiempo de permanencia en un país donde se hable la lengua meta inciden en la adquisición de la competencia pragmática, aunque también deben tomarse en cuenta otros factores sociales y psicológicos de los aprendices para lograr una explicación más global y coherente.

10. Estas no son percepciones antojadizas mías al evaluar los enunciados sino que surgen de la comparación con respuestas dadas por nativos a la misma situación. Al respecto, véase mi trabajo titulado "Percepción de la cortesía a partir de la aplicación de un test de hábitos sociales en Costa Rica."

\section{Referencias bibliográficas}

Austin, J. How to Do Things with Words. Oxford: Oxford University Press, 1962.

Bardovi-Harlig. "Empirical evidence of the need for instruction in pragmatics." En Rose, K. y Kasper, G. (eds). Pragmatics in Language Teaching. Nueva York: Cambridge University Press. 2001.

Bravo, Diana. ¿Imagen "positiva” vs. Imagen "negativa"?: pragmática sociocultural y componentes de face." Oralia. Análisis del discurso oral. Vol. 2. 1999.

Brown, P. Levinson, S. Politeness, some universal in language use. Cambridge. Cambridge University Press

Cook, Vivian. Second Language Learning and Language Teaching. Nueva York: Oxford University Press. 2001.

Crystal, David (Ed.). The Cambridge encyclopedia of language. Nueva York: Cambridge University Press. 1997.

Escandell Vidal, M.V. Introducción a la pragmática. Barcelona: Ariel Lingüística. 1996.

Fraser, Bruce. "Perspectives on Politeness". Journal of Pragmatics. Vol. $14,1990$.

Grice, H.P. "Logic and conversation". Cole, P. y Morgan (eds). Syntax and Semantics. Nueva Cork: Academy Press. 1975. 
Harper, Jeremy. The practice of English Language Teaching. Nueva York: Longman. 1983.

Haverkate, Henk. La cortesía verbal. Estudio pragmalingüístico. Madrid: Gredos. 1994.

Kasper, G. "Data collection in Pragmatics research". Culturally Speaking: managing rapport through talk across cultures. Spencer-Oatey, (ed.). New York: Continuum. 2000.

Kasper, G. y Rose, K. Pragmatic development in a Second Language. Language Learning. Vol. 52. 2002.

Klein, Wolfgang. Second Language Acquisition. Nueva York: Cambridge University Press. 1986.

Lakoff, R. "The logic of politeness; or, minding your p's and q's". Papers from the Regional Meeting. Chicago Linguistic Society, IX, 1973.

Larsen Freeman, D. y Long, M. Introducción al estudio de adquisición de segundas lenguas. Madrid: Gredos. 1994.

Mao, R.L. "Beyond politeness theory, "face" revisited and renewed." Journal of Pragmatics, 21. 1994.

Matsumoto, Y. "Reexamination of the universality of face. Politeness phenomena in Japanese. Journal of Pragmatics, 12. 1988.

Murillo, Jorge. "La cortesía verbal en el español de Costa Rica.”. Revista Káñina. Vol. XXVI N. 2. 2002.

" "La cortesía verbal en situaciones de habla en Costa Rica: hacia la comprensión de la imagen social en su contexto sociocultural." Estudios del discurso de cortesía en español. I coloquio del programa EDICE. Diana Bravo (ed.), Estocolmo. 2003.

- "Percepción de la cortesía a partir de la aplicación de un test de hábitos sociales en Costa Rica." (En prensa). 2003.

. "La cortesía verbal en Costa Rica. Percepciones de los hablantes sobre la (des)cortesía en puestos de atención al público." Pragmática sociocultural: estudios sobre el discurso de cortesía en español. Diana Bravo y Antonio Briz (eds.). Barcelona: Editorial Ariel. 2004.

Muñoz, Carmen. Segundas lenguas. Adquisición en el aula. Barcelona: Ariel. 2000.

Omaggio, Alice. Teaching Language in Context. Boston: Heinle and Heinle Publishers. 1993.

Piatti, Guillermina. "La elaboración de test de hábitos sociales para la enseñanza del español como lengua extranjera." Estudios del discurso de cortesía en español. I coloquio del programa EDICE. Diana Bravo (ed.), Estocolmo. 2003.

Rivers, W., Azevedo, M. y Heflin, W. Teaching Spanish. A practical Guide. Chicago: National Textbook Co. 1994.

Sánchez, Aquilino. Los métodos en la enseñanza de idiomas. Madrid: Sociedad General Española de Librería S. A. 1997.

Schmidt, R. "Consciousness, learning and interlanguage pragmatics". En Kasper, G. y Blum-Kulka, S. Interlanguage Pragmatics. Oxford: Oxford University Press. 1993. 
Schriffin, Deborah. Approaches to discourse. Cambridge: Blacwell. 1994.

Savignon, Sandra. Commucative Competence: Theory and Classroom Practice. Reading, Masachussets: Addison-Wesley. 1983.
Searle, J.R. "Indirect Speech Acts". Cole, P. y Morgan (eds). Syntax and Semantics. Nueva Cork: Academy Press. 1975.

Srum, J. y Glisan, E. Teacher's Handbook. Contextualized Language Instruction. Boston: Heinle and Heinle Publishers. 1994. 